\title{
Associations of hospital characteristics with nosocomial pneumonia after cardiac surgery can impact on standardized infection rates
}

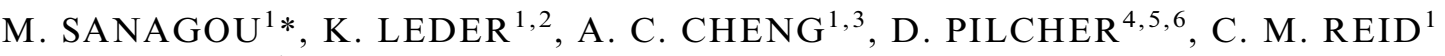 \\ AND R. WOLFE ${ }^{1}$ \\ ${ }^{1}$ Department of Epidemiology and Preventive Medicine, Faculty of Medicine, Nursing and Health Sciences, \\ Monash University, Melbourne, VIC, Australia \\ ${ }^{2}$ Victorian Infectious Diseases Service, Royal Melbourne Hospital, Melbourne, VIC, Australia \\ ${ }^{3}$ Department of Infectious Diseases, Alfred Health, Melbourne, VIC, Australia \\ ${ }^{4}$ Department of Intensive Care Medicine, The Alfred Hospital, Melbourne, VIC, Australia \\ ${ }^{5}$ The Australian and New Zealand Intensive Care Research Centre, Department of Epidemiology and Preventive \\ Medicine, Monash University, Melbourne, VIC, Australia \\ ${ }^{6}$ The Australian and New Zealand Intensive Care Society (ANZICS) Centre for Outcome and Resource \\ Evaluation, Ievers Terrace, Carlton, VIC, Australia
}

Received 14 May 2015; Final revision 7 September 2015; Accepted 8 September 2015; first published online 9 October 2015

\section{SUMMARY}

To identify hospital-level factors associated with post-cardiac surgical pneumonia for assessing their impact on standardized infection rates (SIRs), we studied 43691 patients in a cardiac surgery registry (2001-2011) in 16 hospitals. In a logistic regression model for pneumonia following cardiac surgery, associations with hospital characteristics were quantified with adjustment for patient characteristics while allowing for clustering of patients by hospital. Pneumonia rates varied from $0 \cdot 7 \%$ to $12 \cdot 4 \%$ across hospitals. Seventy percent of variability in the pneumonia rate was attributable to differences in hospitals in their long-term rates with the remainder attributable to within-hospital differences in rates over time. After adjusting for patient characteristics, the pneumonia rate was found to be higher in hospitals with more registered nurses (RNs)/100 intensive-care unit (ICU) admissions [adjusted odds ratio (aOR) 1·2, $P=0 \cdot 006$ ] and more RNs/available ICU beds (aOR 1·4, $P<0 \cdot 001$ ). Other hospital characteristics had no significant association with pneumonia. SIRs calculated on the basis of patient characteristics alone differed substantially from the same rates calculated on the basis of patient characteristics and the hospital characteristic of RNs/100 ICU admissions. Since SIRs using patient case-mix information are important for comparing rates between hospitals, the additional allowance for hospital characteristics can impact significantly on how hospitals compare.

Key words: Pneumonia.

\section{INTRODUCTION}

Reducing complications after cardiac surgery should improve patient outcomes and save the health system considerable expense. Pneumonia incidence in patients who have undergone heart surgery may reach $20 \%$, and carries a strong association with subsequent mortality

\footnotetext{
* Author for correspondence: M. Sanagou, PhD, Department of Epidemiology and Preventive Medicine, School of Public Health and Preventive Medicine, Monash University, Alfred Hospital, Commercial Rd, Melbourne, VIC 3004, Australia.

(Email: Masoumeh.Sanagou@monash.edu)
} 
$[1,2]$. Age, emergency surgery, chronic obstructive pulmonary disease (COPD), and smoking have been identified as patient-related factors strongly associated with the occurrence of pneumonia after cardiac surgery [2-5].

It is clear that infection rates vary across hospitals, but whether hospital-level characteristics are associated with pneumonia risk following cardiac surgery remains unclear. This may be due to differences in measurement of nurse staffing [e.g. registered nurse (RN) hours or RN proportion], sources of data on hospital-level characteristics, different research designs (e.g. cross-sectional $v s$. longitudinal), different statistical approaches to data analysis, or different patient samples (medical patients, surgical patients, mixed medical and surgical patients) [6-17]. However, some hospital-level variables remain entirely unexplored despite much literature suggesting that certain hospital characteristics relate to poorer patient outcomes in general.

In this context, we sought to understand better whether hospital characteristics such as hospital volume, number of hospital beds, RN staffing, standards for airway management, standards for central line insertion, and rounds with an infectious disease specialist are associated with pneumonia following cardiac surgery. Further we aimed to assess whether such associations, if they exist, could impact on standardized infection rates (SIRs) and hence alter hospital comparisons.

\section{METHODS}

\section{Data source and study population}

This study used information from the Australian and New Zealand Society of Cardiac and Thoracic Surgeons (ANZSCTS) registry of cardiac surgery procedures from 2001 to 2011 [18]. All patients were treated post-operatively in an intensive-care unit (ICU) for at least $1 \mathrm{~h}$. This project was undertaken following approval from the ANZSCTS Research Committee, which governs access to data from the registry. Ethical approval for the use of de-identified registry data for secondary research purposes had previously been provided by each participating institution's ethics review committee.

The Australian and New Zealand Intensive Care Society-Centre for Outcome and Resource Evaluation (ANZICS-CORE) has collected audit and analysis data on performance indicators of Australian and New Zealand ICUs since 1992. ANZICS-CORE provided hospital-level characteristics from 2001 to 2011 annual surveys after approval from each ICU director of participating hospitals in the ANZSTCS registry [19]. For the hospital-level variables related to measures of ICU staffing, missing values were replaced by one of the following methods as appropriate: (i) the average of 1 year before and 1 year after the year with missing information, (ii) the average of the two preceding annual values, (iii) the average of the available information.

For the hospital-level variables related to measures of ICU safety and quality, the 2007 values were used as these represented approximately a mid-point in study duration given the later entry of some of the hospitals to the registry.

\section{Outcome measures}

Data on three relevant post-operative syndromes within 30 days of surgery were collected in the ANZSCTS registry:

(1) Pneumonia. Diagnosed by one of the following: positive non-quantitative cultures of sputum or trans-tracheal aspirate with a recognized pathogen and clinical and radiological features consistent with pneumonia with onset after surgery.

(2) Ventilation. Post-operative pulmonary insufficiency requiring ventilatory support-including (but not limited to) causes such as acute respiratory distress syndrome (ARDS) and pulmonary oedema - for a total period of $>24 \mathrm{~h}$.

(3) Re-intubation. Was re-intubation required for any reason during hospitalization?

Due to the lack of a clear gold standard, three definitions of pneumonia were used based on the three syndromes as follows: (i) pneumonia, (ii) pneumonia and ventilation, and (iii) pneumonia and ventilation and re-intubation. Definition (i) 'pneumonia', was treated as the main outcome in subsequent analyses but results are also presented for definitions (ii) and (iii).

\section{Patient-level variables}

An existing prediction model for nosocomial pneumonia after cardiac surgery [20] which was validated for use in the Australian setting [21] was used to adjust for patient-level variables (Table 1). Using this defined prediction tool avoided the subjectivity involved in selecting variables for inclusion in our analysis. The prediction tool combined information on underweight status [body mass index $(\mathrm{BMI})<18 \cdot 5$, where BMI was calculated as weight $(\mathrm{kg})$ divided by height $\left(\mathrm{m}^{2}\right)$ ], smoking history defined as having smoked cigarettes 


\begin{tabular}{|c|c|c|c|c|c|c|}
\hline \multirow[b]{2}{*}{ Risk factor } & \multicolumn{2}{|c|}{$\begin{array}{l}\text { All patients, } \\
(N=43691)\end{array}$} & \multicolumn{2}{|c|}{$\begin{array}{l}\text { Patients with } \\
\text { pneumonia } \\
(N=2229)\end{array}$} & \multicolumn{2}{|c|}{$\begin{array}{l}\text { Patients without } \\
\text { pneumonia } \\
(N=41462)\end{array}$} \\
\hline & $n$ & $\%$ & $n$ & $\%$ & $n$ & $\%$ \\
\hline Underweight, BMI <18.5 & 543 & $1 \cdot 2$ & 44 & $2 \cdot 0$ & 499 & $1 \cdot 2$ \\
\hline Smoking history & 27122 & $62 \cdot 1$ & 1556 & $69 \cdot 8$ & 25566 & $61 \cdot 7$ \\
\hline COPD & 1648 & $3 \cdot 8$ & 132 & $5 \cdot 9$ & 1516 & $3 \cdot 7$ \\
\hline CCS class $\geqslant 3$ & 14545 & $33 \cdot 3$ & 818 & $36 \cdot 7$ & 13727 & $33 \cdot 1$ \\
\hline Creatinine level $>1.2 \mathrm{mg} / \mathrm{dl}$ & 10867 & $24 \cdot 9$ & 773 & $34 \cdot 7$ & 10094 & $24 \cdot 3$ \\
\hline Emergent surgery & 2071 & $4 \cdot 7$ & 278 & $12 \cdot 5$ & 1793 & $4 \cdot 3$ \\
\hline Intraoperative blood transfusion & 19144 & $43 \cdot 8$ & 1556 & $69 \cdot 8$ & 17588 & $42 \cdot 4$ \\
\hline PTCA during hospitalization & 406 & $0 \cdot 9$ & 36 & $1 \cdot 6$ & 370 & $0 \cdot 9$ \\
\hline Mechanical ventilation $>1$ day & 5664 & $13 \cdot 0$ & 1015 & $45 \cdot 5$ & 4649 & $11 \cdot 2$ \\
\hline
\end{tabular}

BMI, Body mass index; COPD, chronic obstructive pulmonary disease; CCS class, Canadian cardiovascular society classification of angina; PTCA, percutaneous transluminal coronary angioplasty.

BMI calculated as weight $(\mathrm{kg})$ divided by height $\left(\mathrm{m}^{2}\right)$.

during the year before hospital admission, COPD, Canadian Cardiovascular Society classification of angina [22] (CCS) class $\geqslant 3$, serum creatinine level (the most recent measurement) $>1.2 \mathrm{mg} / \mathrm{dl}(106.1 \mathrm{mmol} / \mathrm{ml})$, surgery urgency categorized as non-emergency and emergency, intraoperative blood transfusion, percutaneous transluminal coronary angioplasty (PTCA) during hospitalization and mechanical ventilation $>1$ day.

\section{Hospital-level variables}

Existing literature on hospital-level associations with development of pneumonia after cardiac surgery indicated potential importance of measures of hospital size, measures of ICU patient load and measures of ICU safety and quality [10, 13-17].

\section{Measures of hospital size}

Hospital volume was calculated for each hospital from the ANZSCTS registry as the median of the hospital's annual number of cardiac surgeries across 2001-2011. All subsequent hospital-level factors were as reported in the ANZICS-CORE annual survey. Hospital bed number was the total number of beds in each hospital. This is self-reported by hospitals to the ANZICS Critical Care Resources (CCR) registry and represents the number of in-patient hospital beds (excluding beds for psychiatry and day case procedures).

\section{Measures of ICU staffing}

RNs/100 ICU admissions was defined as 100 times the number of RNs full-time equivalent staff divided by total ICU admissions. RNs/available ICU bed was defined as the number of RNs full-time equivalent staff divided by total available ICU beds (including cardiothoracic ICU beds). An available ICU bed is a bed in use or immediately available, which has advanced life support capability and is fully staffed and funded.

\section{Measure of ICU safety and quality}

As part of the annual ANZICS-CORE survey, hospitals are asked if they have processes and standards for accreditation and management of various procedures and processes within the ICU. Markers of hospital safety and quality that were reported included those that met standards for airway management, met standards for central line insertion, and conducted routine rounds with an infectious disease specialist; all were dichotomous variables (yes/no).

\section{Statistical analysis}

An updated version of an existing prediction model for nosocomial pneumonia after cardiac surgery [20] was applied to the ANZSCTS dataset [21]. The updated version of the model coefficients yielded reasonable predictive ability as indicated by the receiveroperating characteristic curve $(\mathrm{AUC})=0 \cdot 71$, and was well calibrated [21]. 


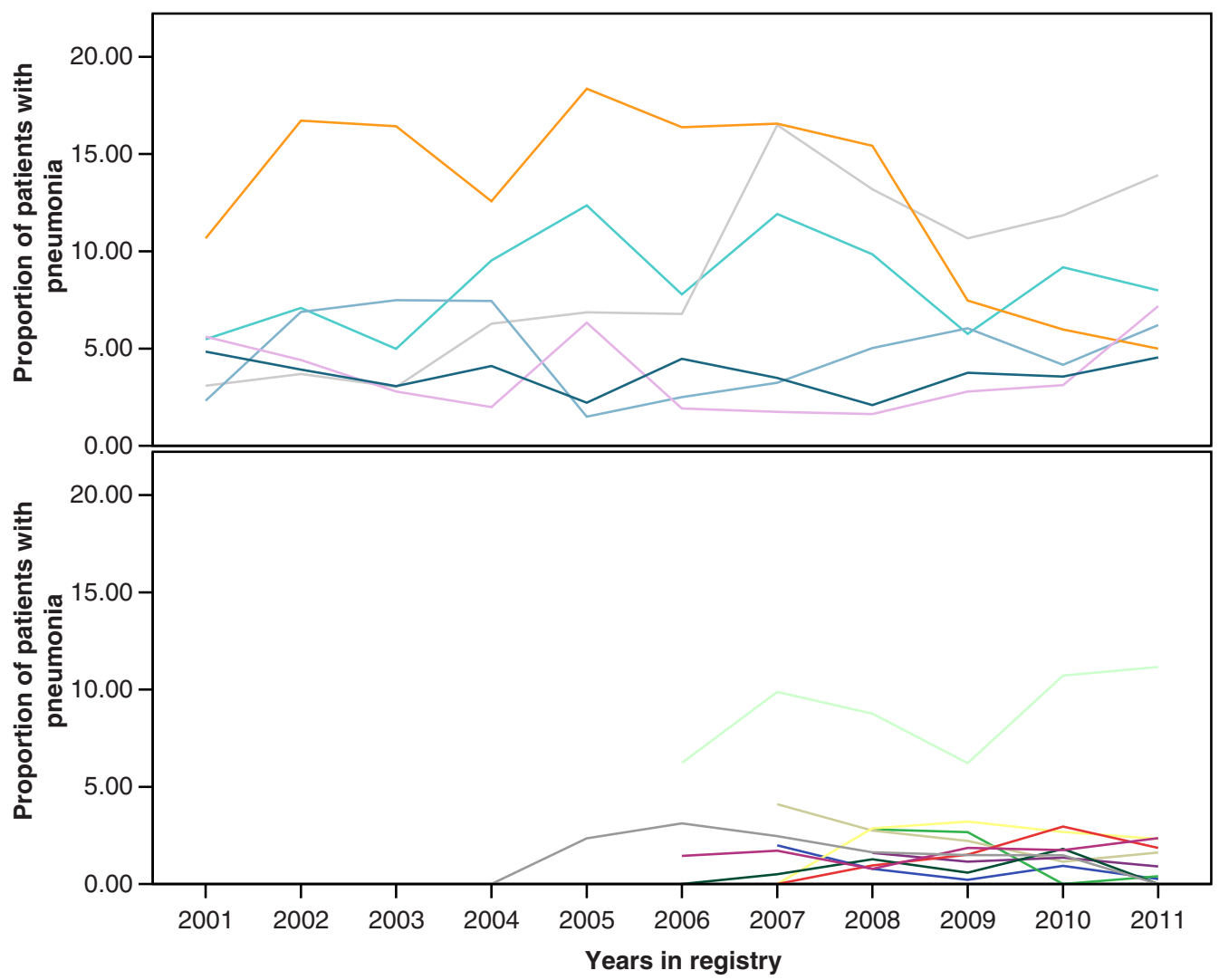

Fig. 1. The nosocomial pneumonia rate (\%) in 16 hospitals between 2001 and 2011. Top panel shows six hospitals present in the registry from its inception, 2001. Bottom panel shows 10 hospitals that joined the registry in later years.

Intra-class correlation (ICC) was used to measure the total variability in pneumonia incidence between hospitals compared to within hospitals over the study period. To address potential within-hospital correlation, a logistic regression model using generalized estimating equations (GEEs) with an exchangeable working correlation structure was fitted to nosocomial pneumonia. This allowed for within-hospital clustering of patient-level responses [23]. In the GEE approach, the coefficient for each hospital-level variable is an estimated population-averaged relationship between the variable and patient-level outcome, adjusting for any patient-level characteristics included in the model.

SIRs were calculated as the pneumonia rate observed in the hospital of interest (i.e. observed) divided by the pneumonia rate in a standard population (i.e. expected) and were used to compare the pneumonia rates in hospitals. Hospitals with SIR $<1$ (number of observed pneumonia cases following cardiac surgery is less than predicted) are 'better than expected' and hospitals with SIR $>1$ are 'worse than expected'.

Statistical significance was defined as $P<0.05$ with no adjustment for multiple hypothesis testing. All statistical analyses were performed using Stata v. 11 (StataCorp., USA).

\section{RESULTS}

In total, 54217 cardiac operations were performed in 25 hospitals during the 11 -year study period. Nine hospitals (6540 patients) were excluded either because they were in the registry $<1$ year (seven hospitals), did not have adult patients (one hospital) or because they declined to have their ANZICS-CORE hospital-level data released (one hospital). In the remaining 16 hospitals, $3986(8 \cdot 4 \%)$ patients were excluded due to missing data for outcome, patient-level, or hospital-level variables.

\section{Characteristics of patients and hospitals}

In the remaining 43691 patients the mean \pm standard deviation (s.D.) age was $66 \pm 13$ years. Nosocomial pneumonia was reported in $5 \cdot 1 \%(n=2229)$, varying from $0 \cdot 7 \%$ to $12 \cdot 4 \%$ across hospitals and overall from $3 \cdot 7 \%$ to $6 \cdot 9 \%$ between 2001 and 2011 (Fig. 1). 
For patients with pneumonia, 30-day mortality was $7 \cdot 1 \%$ and mean duration of ventilation was $104 \pm 222 \mathrm{~h}$, compared to a 30-day mortality rate of $2 \cdot 1 \%$ and mean $21 \pm 60 \mathrm{~h}$ ventilation for patients who did not have pneumonia.

Table 1 summarizes the characteristics of the study population.

Hospital volume was on average $428 \pm 101$ (range 183-573) surgeries, number of hospital beds averaged $448 \pm 155$ (range 118-860) beds and the mean number of full-time equivalent RNs was $102 \pm 50$ (range 9223). In $29 \%$ of hospitals, rounds with an infectious disease specialist were provided; $44 \%$ and $38 \%$ of hospitals met standards for airway management and central line insertion, respectively.

\section{Between-hospital variability in pneumonia incidence}

An ICC of $0 \cdot 70$ [95\% confidence interval (CI) 0.5-0.9] suggests that $70 \%$ of the total variability in pneumonia incidence is explained by differences between hospitals, with the remainder attributable to within-hospital differences over time. In other words, the changes in pneumonia incidence within a hospital over the 10 years were minor in comparison to the differences between hospitals in their respective long-term pneumonia incidence rates.

In unadjusted analysis, the ratio of $\mathrm{RNs} / 100 \mathrm{ICU}$ admissions [odds ratio (OR) $1 \cdot 2,95 \%$ CI $1 \cdot 1-1 \cdot 3$, $P<0.001]$ and the ratio of RNs/available ICU bed (OR 1.5, 95\% CI 1·2-1·7, $P<0 \cdot 001$ ) were associated with pneumonia incidence. Indicating increased incidence rates being accompanied by higher nurse staffing levels/100 ICU admissions and per available ICU bed, this relationship is illustrated in Figure 2.

The results of univariate analysis, and multivariate analysis after adjusting for patient-level characteristics, are described in Table 2. Other hospital-level characteristics including hospital volume, number of hospital beds, standards for airway management, standards for central line insertion, and rounds with an infectious disease specialist did not exhibit any significant association with pneumonia incidence.

After adjusting for patient-level variables, the risk of pneumonia was still higher in hospitals with more RNs/100 ICU admissions (adjusted OR 1·2, 95\% CI $1 \cdot 0-1 \cdot 3, \quad P=0 \cdot 006)$ and more RNs/available ICU bed (adjusted OR 1·4, 95\% CI 1·2-1·7, $P<0 \cdot 001$ ). The two other definitions of nosocomial pneumonia gave results that were similar to those already presented with no changes in conclusions about hospital-level risk factors. (Table 2)

\section{Standardized infection rates}

Figure 3 shows the change to each hospital's ratio of observed to predicted pneumonia when using a model with patient-level risk factors only to a model that also includes RNs/100 ICU admissions (hospitallevel risk factor). Assessment of whether a hospital's infection rate is as expected alters substantially when 'as expected' takes a hospital-level covariate into account. The hospital-level characteristic holds considerable sway in explaining between-hospital variability in infection rates and hence in explaining SIRs that deviate from SIR $=1$. The effect is to bring the majority of hospital SIRs towards SIR $=1$.

\section{DISCUSSION}

This study found some evidence that hospital characteristics are related to variations in the incidence of nosocomial pneumonia after cardiac surgery and that accounting for these characteristics can have a major impact on comparisons of hospital performance via standardized pneumonia rates.

Across the 43000 patients from 16 Australian hospitals in this study, pneumonia incidence rates varied considerably. The development of pneumonia after cardiac surgery was found to be associated with two hospital characteristics although the direction of the association was counterintuitive; pneumonia risk was found to be positively associated with the number of $\mathrm{RNs} / 100$ ICU admissions and per available ICU bed. This association was evident in both crude and adjusted analyses. It is possible that higher staffing levels are a surrogate marker for an unmeasured confounder (e.g. higher risk patients not identified by other measures), or that increasing rates of postoperative pneumonia require higher levels of nurse staffing in the ICU.

Given that pneumonia is a nurse-sensitive outcome $[14,24]$, ICU nurses have a key role in preventing pneumonia in most patients [24]. Our study found an association between pneumonia and one of the nurse staffing measures but not with another. This can be added to the inconsistent evidence of association between nursing ratios and pneumonia [25] In European settings, a Belgian study [13] showed no association between nurse staffing and pneumonia, but Swiss [26] and British [27] studies found an inverse 

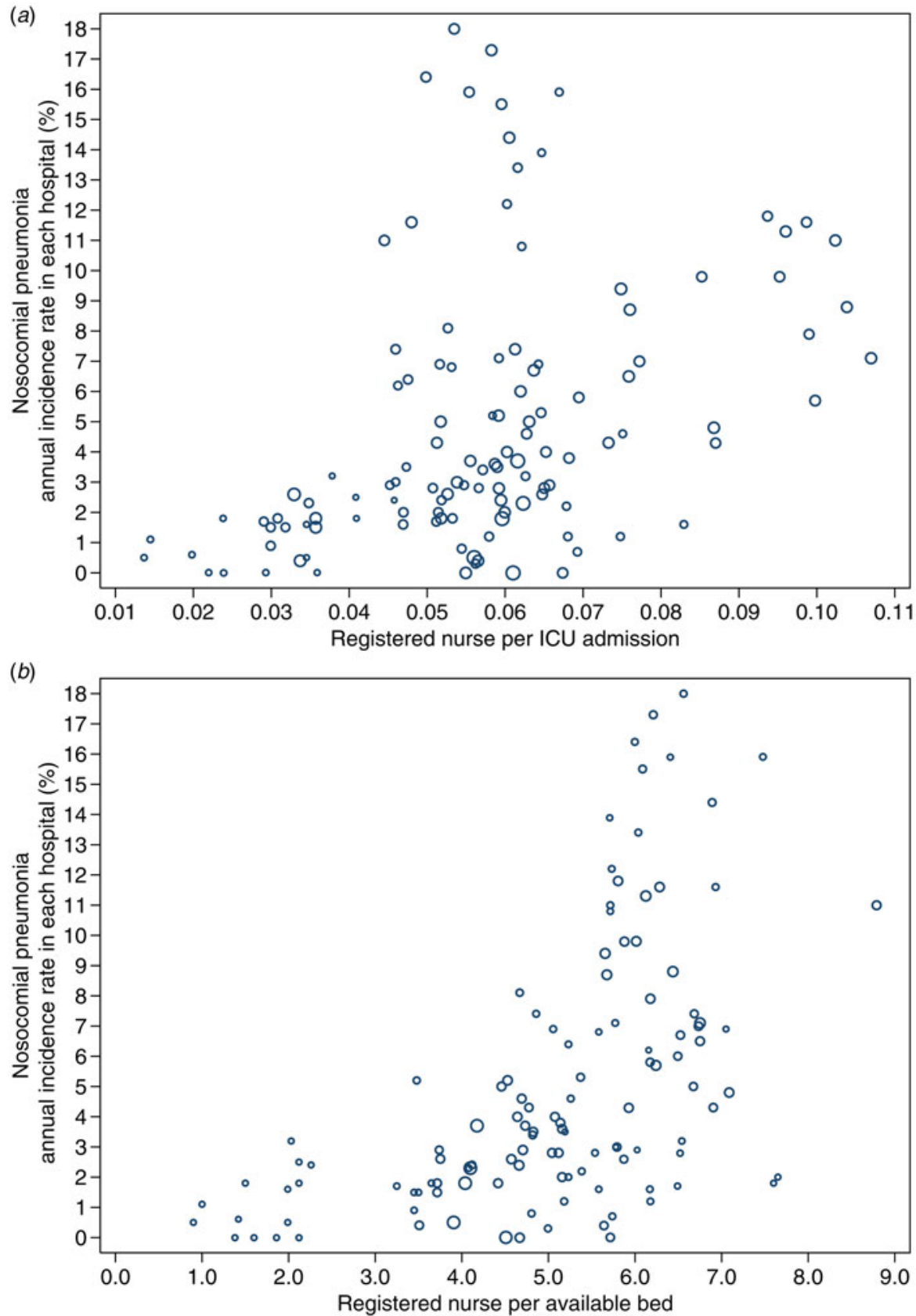

Fig. 2. The relationship between nosocomial pneumonia annual cumulative incidence rate (\%) and number of registered nurses/ICU admissions and per available intensive-care unit (ICU) bed in the corresponding year. The area of each circle is proportional to hospital size as measured by the hospital's $(a)$ total number of ICU admission and $(b)$ total number of available beds in the given year, respectively.

relationship. In American settings [6, 7, 28] an inverse relationship between nurse staffing and pneumonia was found in California hospitals but not in New York hospitals. This relationship was reported in surgical and medical units [8]. While an inverse relationship between the number of RNs/patient days and pneumonia has been found after surgery but not after invasive vascular procedures [29], this relationship was not confirmed in other studies $[9,11,30]$. Our finding of a positive association differs from most other studies. It is likely that a high nursing:patient ratio is a surrogate marker for ICUs that treat more complex patients. It may also represent an effect rather than a cause, and indicates the stress on resources resulting from higher rates of this complication over the long term. 
Table 2. The results of univariate and multivariate analysis after adjusting for patient-level characteristics

\begin{tabular}{|c|c|c|c|c|c|c|}
\hline \multirow[b]{2}{*}{ Hospital characteristics } & \multicolumn{3}{|c|}{ Univariate analysis } & \multicolumn{3}{|c|}{$\begin{array}{l}\text { Multivariate analysis adjusting } \\
\text { for patient-level risk factors }\end{array}$} \\
\hline & OR & $(95 \% \mathrm{CI})$ & $P$ & OR & $(95 \% \mathrm{CI})$ & $P$ \\
\hline \multicolumn{7}{|l|}{ Outcome: definition (i) ( $n=2229$ cases) } \\
\hline Hospital volume (/100 surgeries) & $0 \cdot 9$ & $(0 \cdot 6-1 \cdot 3)$ & $0 \cdot 5$ & $0 \cdot 9$ & $(0 \cdot 7-1 \cdot 3)$ & $0 \cdot 6$ \\
\hline Hospital beds (/100 beds) & $1 \cdot 1$ & $(0 \cdot 9-1 \cdot 3)$ & $0 \cdot 4$ & $1 \cdot 1$ & $(0 \cdot 9-1 \cdot 3)$ & $0 \cdot 4$ \\
\hline RNs/100 ICU admissions & $1 \cdot 2$ & $(1 \cdot 1-1 \cdot 3)$ & $<0 \cdot 001$ & $1 \cdot 2$ & $(1 \cdot 0-1 \cdot 3)$ & $0 \cdot 006$ \\
\hline RNs/available ICU bed & $1 \cdot 5$ & $(1 \cdot 2-1 \cdot 7)$ & $<0 \cdot 001$ & $1 \cdot 4$ & $(1 \cdot 2-1 \cdot 7)$ & $<0 \cdot 001$ \\
\hline Standards for airway management (yes) & $1 \cdot 2$ & $(0 \cdot 5-2 \cdot 7)$ & $0 \cdot 6$ & $1 \cdot 2$ & $(0 \cdot 6-2 \cdot 4)$ & $0 \cdot 6$ \\
\hline Standards for central line insertion (yes) & $1 \cdot 6$ & $(0 \cdot 8-3 \cdot 4)$ & $0 \cdot 2$ & $1 \cdot 7$ & $(0 \cdot 9-3 \cdot 4)$ & $0 \cdot 1$ \\
\hline Rounds with infectious disease specialist (yes) & $1 \cdot 0$ & $(0 \cdot 4-2 \cdot 4)$ & $0 \cdot 9$ & $1 \cdot 1$ & $(0 \cdot 5-2 \cdot 2)$ & $0 \cdot 8$ \\
\hline \multicolumn{7}{|l|}{ Outcome: definition (ii) ( $n=1176$ cases) } \\
\hline Hospital volume (/100surgeries) & $1 \cdot 0$ & $(0 \cdot 7-1 \cdot 4)$ & $0 \cdot 8$ & $1 \cdot 0$ & $(0 \cdot 7-1 \cdot 4)$ & $0 \cdot 9$ \\
\hline Hospital beds (/100 beds) & $1 \cdot 0$ & $(0 \cdot 8-1 \cdot 2)$ & $0 \cdot 9$ & $1 \cdot 0$ & $(0 \cdot 8-1 \cdot 2)$ & $0 \cdot 8$ \\
\hline RNs/100 ICU admissions & $1 \cdot 2$ & $(1 \cdot 1-1 \cdot 3)$ & $<0 \cdot 001$ & $1 \cdot 1$ & $(1 \cdot 0-1 \cdot 2)$ & $0 \cdot 02$ \\
\hline RNs/available ICU bed & $1 \cdot 4$ & $(1 \cdot 2-1 \cdot 6)$ & $<0 \cdot 001$ & $1 \cdot 3$ & $(1 \cdot 1-1 \cdot 6)$ & $0 \cdot 001$ \\
\hline Standards for airway management (yes) & $1 \cdot 3$ & $(0 \cdot 5-3 \cdot 2)$ & $0 \cdot 5$ & $1 \cdot 3$ & $(0 \cdot 6-2 \cdot 8)$ & $0 \cdot 4$ \\
\hline Standards for central line insertion (yes) & $1 \cdot 5$ & $(0 \cdot 6-3 \cdot 6)$ & $0 \cdot 3$ & $1 \cdot 7$ & $(0 \cdot 8-3 \cdot 3)$ & $0 \cdot 1$ \\
\hline Rounds with infectious disease specialist (yes) & $1 \cdot 1$ & $(0 \cdot 4-3 \cdot 0)$ & $0 \cdot 9$ & $1 \cdot 1$ & $(0 \cdot 5-2 \cdot 6)$ & $0 \cdot 8$ \\
\hline \multicolumn{7}{|l|}{ Outcome: definition (iii) ( $n=501$ cases) } \\
\hline Hospital volume (/100 surgeries) & $1 \cdot 0$ & $(0 \cdot 8-1 \cdot 3)$ & $0 \cdot 9$ & $1 \cdot 1$ & $(0 \cdot 8-1 \cdot 3)$ & $0 \cdot 6$ \\
\hline Hospital beds (/100 beds) & $0 \cdot 9$ & $(0 \cdot 8-1 \cdot 1)$ & $0 \cdot 6$ & $0 \cdot 9$ & $(0 \cdot 8-1 \cdot 1)$ & $0 \cdot 6$ \\
\hline RNs/100 ICU admissions & $1 \cdot 2$ & $(1 \cdot 1-1 \cdot 2)$ & $<0 \cdot 001$ & $1 \cdot 1$ & $(1 \cdot 0-1 \cdot 1)$ & $0 \cdot 008$ \\
\hline RNs/available ICU bed & $1 \cdot 3$ & $(1 \cdot 2-1 \cdot 5)$ & $<0 \cdot 001$ & $1 \cdot 2$ & $(1 \cdot 1-1 \cdot 4)$ & $<0 \cdot 001$ \\
\hline Standards for airway management (yes) & $1 \cdot 2$ & $(0 \cdot 6-2 \cdot 6)$ & $0 \cdot 6$ & $1 \cdot 1$ & $(0 \cdot 7-2 \cdot 2)$ & $0 \cdot 6$ \\
\hline Standards for central line insertion (yes) & $1 \cdot 1$ & $(0 \cdot 5-2 \cdot 6)$ & $0 \cdot 8$ & $1 \cdot 3$ & $(0 \cdot 8-2 \cdot 2)$ & $0 \cdot 3$ \\
\hline Rounds with infectious disease specialist (yes) & $1 \cdot 1$ & $(0 \cdot 5-2 \cdot 4)$ & $0 \cdot 9$ & $1 \cdot 1$ & $(0 \cdot 6-2 \cdot 1)$ & $0 \cdot 6$ \\
\hline
\end{tabular}

OR, Odds ratio; $\mathrm{CI}$, confidence interval; $\mathrm{RN}$, registered nurse; ICU, intensive care unit;

Definition (i): Pneumonia; definition (ii): pneumonia and ventilation; definition (iii): pneumonia and ventilation and re-intubation. Pneumonia diagnosed by one of the following: positive non-quantitative cultures of sputum or trans-tracheal aspirate with a recognized pathogen and clinical and radiological features consistent with pneumonia with onset after surgery. Ventilation: Post-operative pulmonary insufficiency requiring ventilatory support - including (but not limited to) causes such as acute respiratory distress syndrome (ARDS) and pulmonary oedema - for a total period of longer than $24 \mathrm{~h}$. Re-intubation: Was re-intubation required for any reason during hospitalization?

The SIR is a patient safety metric developed by the Centres for Disease Control and Prevention for comparing outcomes across health providers while adjusting for differences in outcome risk [31]. Our results demonstrate that consideration of hospital-level associations with outcome is important when calculating standardized pneumonia rates following cardiac surgery. Additional allowance for a hospital-level association over and above patient characteristics tended to bring SIRs towards 'as expected' performance. This powerful impact calls for incorporation of hospital-level associations, although in our analysis the counterintuitive nature of the association is somewhat problematic, as discussed above. Nevertheless the results are a marked illustration of the potential importance of hospital characteristics in the calculation of SIRs.
Our study has several limitations. Of 26 public and 33 private Australian hospitals that undertake cardiac surgery, 20 public and eight private hospitals are enrolled and participate in the ANZSCTS Cardiac Surgery Database programme. For this study we studied a subset of 13 public and three private hospitals from the registry which may not be representative of all relevant Australian hospitals, which may impact the generalizability of findings. Additionally, even this many hospitals may still have been insufficient to detect associations for binary hospital-level factors such as meeting standards for airway management and central line insertion, and rounds with an infectious disease specialist. Although guidelines for difficult airway management and for central line insertion have been produced by professional societies 


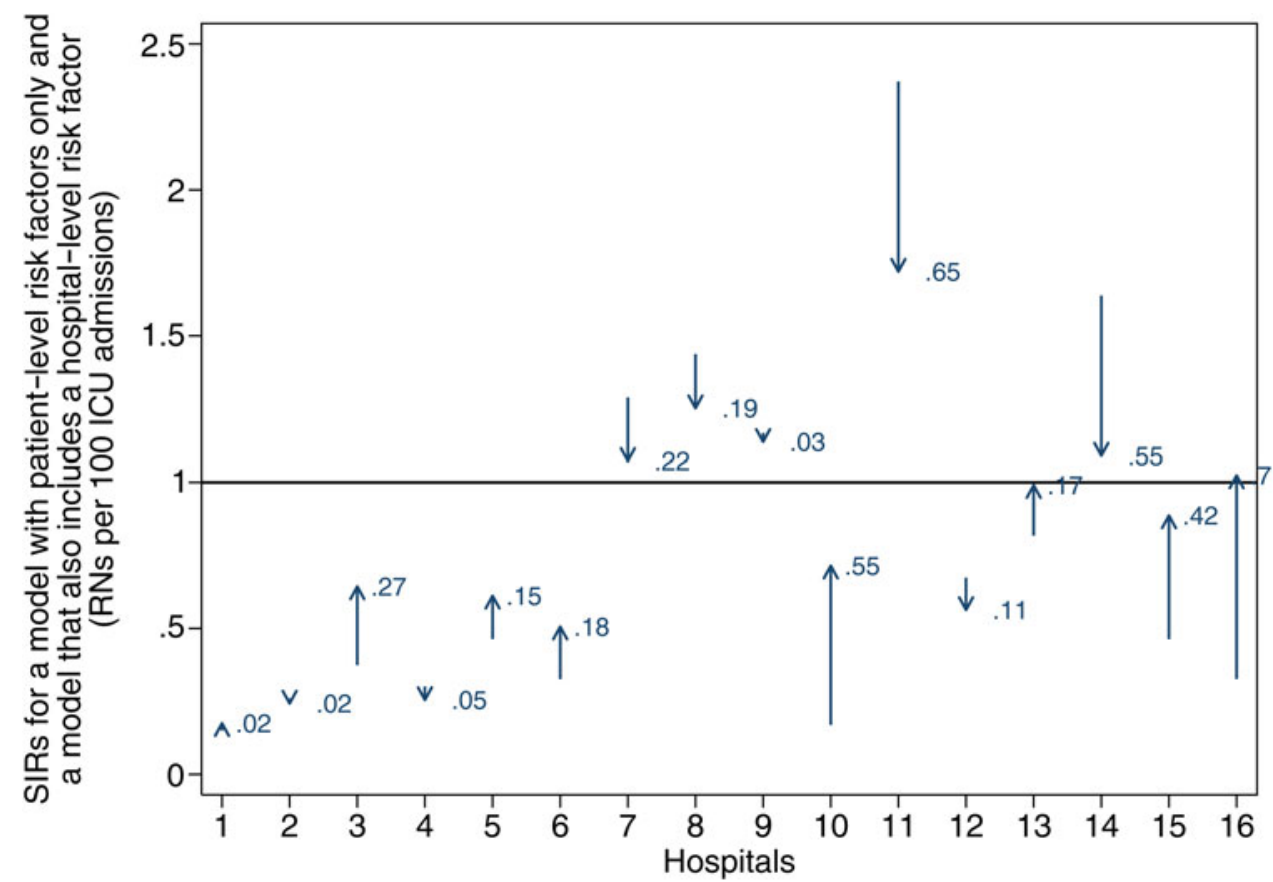

Fig. 3. Standardized infection rates from the ratio of observed to predicted pneumonia for a model with patient-level risk factors only (tail of arrows) and a model that also includes $\mathrm{RNs} / 100$ intensive-care unit (ICU) admissions as a hospital-level risk factor (head of arrows) in 16 hospitals. Examples for comparing the hospital standardized infection rates (SIRs): The relative change for hospital 10 is 0.55 , which means the SIR increased by $55 \%$ after applying the model including RNs/100 ICU admissions as a hospital-level risk factor. The relative change for hospital 11 is -0.65 , which means the SIR decreased by $65 \%$ after applying the model including RNs/100 ICU admissions as a hospital-level risk factor.

(ANZCA airway guideline and Commission/ANZICS guideline for CVC) and some states (NSW central line guideline), their local implementation and potential modification is at the discretion of professionals within the hospital or ICU [32-34]. The ANZICS-CORE annual survey asks if hospitals have a 'standard or policy' for the relevant procedure. Information from the ANZICS-CORE Annual Survey 2007 was predominantly used and some of this hospital-level information may have changed over time. Further, other hospital-level factors of potential importance such as bed occupancy and staffing/1000 patient days were not available.

The North American risk prediction model was an objective way of allowing for patient-level risk factors, avoiding the dangers of selectively adjusting for patient-level risk factors, but may leave residual confounding, with no adjustment made for some patientlevel risks. If patient-level risk factors vary by hospital their effects can then be inadvertently linked with hospital-level risk factors.

The reported pneumonia incidence after cardiac surgery is significantly influenced by the definition used.
An epidemiological definition of nosocomial pneumonia is notoriously difficult, as has been recognized in the recent partial abandonment by the National Healthcare Safety Network (NHSN) of 'ventilatorassociated pneumonia' in favour of the more heterogeneous 'ventilator-associated condition' [35]. In this study, our definitions were constrained by the information collected by the registry and the definition of 'pneumonia and ventilation' most closely corresponds to 'possible ventilator-associated pneumonia' in the new NHSN definitions (not available at the time of our study) [36]. Although the use of quantitative cultures may improve the specificity of the diagnosis of pneumonia, these are not widely available in Australian hospitals. Notably, the presence of pneumonia, as defined in our study, was associated with a significant increase in mortality risk. However, the reliability of this definition is uncertain and we cannot exclude the possibility that differences in sensitivity and specificity between observers may be partially responsible for the observed variation in pneumonia incidence between hospitals.

In this study we examined the relationship between hospital-level characteristics and pneumonia following 
cardiac surgery in the Australian context. We found that pneumonia incidence varies considerably between hospitals. We found limited evidence that hospital factors hold associations with pneumonia incidence rates following cardiac surgery. However, the impact of the hospital-level associations on SIR can be marked.

\section{ACKNOWLEDGEMENTS}

General support was received from: ANZSCTS Data Management Centre, CCRE, Monash University: Professor Chris Reid, Dr Lavinia Tran, Mrs Angela Brennan. ANZSCTS Database Program Steering Committee: $\mathrm{Mr}$ Gil Shardey (Chair), Mr Peter Skillington, $\mathrm{Mr}$ Julian Smith, $\mathrm{Mr}$ Andrew Newcomb, Mr Siven Seevanayagam, Mr Bo Zhang, Mr Hugh Wolfenden, Mr Adrian Pick, Mr Jurgen Passage, A/Prof. Rob Baker, Prof Chris Reid, Dr Lavinia Tran, Mr Andrew Clarke.

The following investigators, data managers and institutions participated in the ASCTS Database: Alfred Hospital: Pick A, Duncan J; Austin Hospital: Seevanayagam S, Shaw M; Cabrini Health: Shardey G; Geelong Hospital: Morteza M, Zhang B, Bright C; Flinders Medical Centre: Knight J, Baker R, Helm J, Canning N; Jessie McPherson Private Hospital: Smith J, Baxter H; Hospital: John Hunter Hospital: James A, Scaybrook S; Lake Macquarie Hospital: Dennett B, Mills M; Liverpool Hospital: French B, Hewitt N; Mater Health Services: Diqer AM, Curtis L; Monash Medical Centre: Smith J, Baxter H; Prince of Wales Hospital: Wolfenden H, Weerasinge D; Royal Melbourne Hospital: Skillington P, Wynne R; Royal North Shore Hospital : Sze D; Royal Perth Hospital: Edwards M, Wright M, Le V. Royal Prince Alfred Hospital: Wilson M, Turner L; Powell, C; Sir Charles Gairdner: Kolybaba M; St George Hospital: Fermanis G, Newbon P; St Vincent's Hospital, VIC: Yii M, Newcomb A, Mack J, Duve K; St Vincent's Hospital, NSW: Spratt P, Hunter T; The Canberra Hospital: Bissaker P, Dean A; Townsville Hospital: Tam R, Farley A; Westmead Hospital: Costa R, Halaka M.

The ANZICS CORE Management Committee: David Pilcher, Peter Hicks, Sue Huckson, Johnny Millar and Justin Williams.

The Australian and New Zealand Society of Cardiac and Thoracic Surgeons (ANZSCTS) National Cardiac Surgery Database Program is funded by the Department of Health Victoria, and the Health Administration Corporation (GMCT) and the Clinical Excellence Commission (CEC), NSW.

\section{DECLARATION OF INTEREST}

None.

\section{REFERENCES}

1. Asensio A, Torres J. Proportion of mortality caused by severe hospital acquired infection in open-heart surgery. Clinical Performance and Quality Health Care 1996; 4: 67-73.

2. Rebollo MH, et al. Nosocomial infections in patients having cardiovascular operations: A multivariate analysis of risk factors. Journal of Thoracic and Cardiovascular Surgery 1996; 112: 908-913.

3. Miholic J, et al. Risk factors for severe bacterial infections after valve replacement and aortocoronary bypass operations: analysis of 246 cases by logistic regression. Annals of Thoracic Surgery 1985; 40: 224-228.

4. Gaynes R, et al. Risk factors for nosocomial pneumonia after coronary artery bypass graft operations. Annals of Thoracic Surgery 1991; 51: 215-218.

5. Leal-Noval SR, et al. Nosocomial pneumonia in patients undergoing heart surgery. Critical Care Medicine 2000; 28: 935-940.

6. Knauf RA, et al. Implementing Nursing's Report Card: a Study of RN Staffing, Length of Stay and Patient Outcomes. Washington: American Nurses Publishing, 1997.

7. Lichtig LK, Knauf RA, Milholland DK. Some impacts of nursing on acute care outcomes. Journal of Nursing Administration 1999; 29: 25-33.

8. Needleman $\mathbf{J}$, et al. Nurse-staffing levels and the quality of care in hospitals. New England Journal of Medicine 2002; 346: 1715-1722.

9. Kovner C, et al. Nurse staffing and post surgical adverse events: an analysis of administrative data from a sample of U.S. hospitals: 1990-1996. Health Services Research 2002; 37: 611-629.

10. Cho SH. Using multilevel analysis in patient and organizational outcomes research. Nursing Research 2003; 52: 61-65.

11. Cho SH, et al. The effects of nurse staffing on adverse events, morbidity, mortality, and medical costs. Nursing Research 2003; 52: 71-79.

12. Mark BA, et al. A longitudinal examination of hospital registered nurse staffing and quality of care. Health Services Research 2004; 39: 279-301.

13. Van den Heede $\mathbf{K}$, et al. Nurse staffing and patient outcomes in Belgian acute hospitals: cross-sectional analysis of administrative data. International Journal of Nursing Studies 2009; 46: 928-939.

14. Dall TM, et al. The economic value of professional nursing. Medical Care 2009; 47: 97-104.

15. Kane RL, et al. The association of registered nurse staffing levels and patient outcomes systematic review and meta-analysis. Medical Care 2007; 45: 1195-204.

16. Agabiti $\mathbf{N}$, et al. The association of socioeconomic disadvantage with postoperative complications after 
major elective cardiovascular surgery. Journal of Epidemiology \& Community Health 2008; 62: 882-889.

17. Sermeus W, Van den Heede K, Vleugels A. Database quality of nursing care AG/01/123. Leuven: Katholieke Universiteit Leuven, 2007, pp. 1-193.

18. Reid CM, et al. Initial twelve months experience and analysis for 2001-2002 from the Australasian Society of Cardiac and Thoracic Surgeons-Victorian database project. Heart, Lung and Circulation 2004; 13: 291297.

19. Stow PJ, et al. Development and implementation of a high-quality clinical database: The Australian and New Zealand Intensive Care Society Adult Patient Database. Journal of Critical Care 2006; 21: 133-141.

20. Kinlin LM, et al. Derivation and validation of a vlinical prediction rule for nosocomial pneumonia after coronary artery bypass graft surgery. Clinical Infectious Diseases 2010; 50: 493-501.

21. Sanagou M, et al. External validation and updating of a prediction model for nosocomial pneumonia after coronary artery bypass graft surgery. Epidemiology and Infection 2014: 142: 540-544.

22. Campeau L. Letter: Grading of angina pectoris. Circulation 1976; 54: 522-523.

23. Sanagou M, et al. Hospital-level associations with 30-day patient mortality after cardiac surgery: a tutorial on the application and interpretation of marginal and multilevel logistic regression. BMC Medical Research Methodology 2012; 12: 28.

24. Murray T. Ventilator-associated pneumonia as a nursesensitive outcome: the role of the clinical nurse specialist in the development and implementation of clinical systems to reduce ventilator-associated pneumonia. Clinical Nurse Specialist 2005; 19: 80.

25. Lankshear AJ, Sheldon TA, Maynard A. Nurse staffing and healthcare outcomes: A systematic review of the international research evidence. Advances in Nursing Science 2005; 28: 163-174.

26. Hugonnet S, Villaveces A, Pittet D. Nurse staffing level and nosocomial infections: Empirical evaluation of the casecrossover and case-time-control designs. American Journal of Epidemiology 2007; 165: 1321-1327.

27. Rafferty AM, et al. Outcomes of variation in hospital nurse staffing in English hospitals: cross-sectional analysis of survey data and discharge records. International Journal of Nursing Studies 2007; 44: 175182.

28. American Nurses' Association. Nurse Staffing and Patient Outcomes in the Inpatient Hospital Setting. Washington, DC: American Nurses Association, 2000.

29. Kovner C, Gergen PJ. Nurse staffing levels and adverse events following surgery in U.S. hospitals. Journal of Nursing Scholarship 1998; 30: 315-321.

30. Unruh L. Licensed nurse staffing and adverse events in hospitals. Medical Care 2003; 41: 142-152.

31. Centers for Disease Control and Prevention. National healthcare-associated infections standardized infection ratio report. Using data reported to the National Healthcare Safety Network (July 2009 through December 2009), 2009 (http://www.cdc.gov/HAI/pdfs/stateplans/ SIR-2010_JunDec2009.pdf). Accessed 8 September 2015.

32. New South Wales Health Policy. Central venous access device insertion and post insertion care (PD2011_060). Sydney: NSW Health, 2011 (http://www0.health.nsw. gov.au/policies/pd/2011/pdf/PD2011_060.pdf). Accessed 8 September 2015.

33. Australian and New Zealand Intensive Care Society. Central line insertion and maintenance guideline. Melbourne; ANZICS Safety and Quality Committee, 2012 (www.clabsi.com.au and http://www.anzics.com.au/ Downloads/ANZICS $\% 20$ Central $\% 20$ Line $\% 20$ Insertion $\%$ 20\&\%20Maintenance $\% 20$ Guideline $\% 20$ April $\% 202012$. pdf). Accessed 8 September 2015.

34. Australian and New Zealand College of Anaesthetists. (2012) PS56 Guidelines on equipment to manage a difficult airway during anaesthesia (http://www.anzca.edu.au/ resources/professional-documents/documents/professionalstandards/pdffiles/PS56-guidelines-on-equipment-to-manage-a-difficult-airwayduring-anaesthesia.pdf). Accessed 8 September 2015.

35. Magill SS, Fridkin SK. Improving surveillance definitions for ventilator-associated pneumonia in an era of public reporting and performance measurement. Clinical Infectious Diseases 2012; 54: 378-380.

36. Horan TC, Andrus M, Dudeck MA. CDC/NHSN surveillance definition of healthcare - associated infection and criteria for specific types of infections in the acute care setting. American Journal of Infection Control 2008; 36: 309-332. 